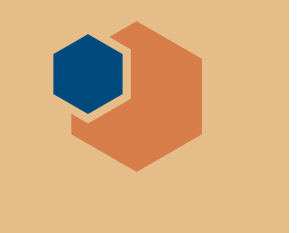

\section{EU Graphene Flagship signals industrial 2D materials}

graphene-flagship.eu
$\mathrm{I}_{\mathrm{B}}^{\mathrm{n}}$ recent years, the 100,000 visitors to Barcelona's Mobile World Congress (MWC), the annual get-together for the mobile telecommunications industry, have been able to see how developments in graphene could "transform future telecommunications.” In February of this year, 26 industrial and academic partners exhibited 20 working demonstrators and prototypes related to graphene at the MWC. These devices, on show in the Graphene Pavilion, focused on "The Phone of the Future," "Wearables of the Future," and "The Home of the Future."

The Graphene Pavilion at MWC is the brainchild of the EU's Graphene Flagship Innovation Team. Supported by the European Commission, the Graphene Flagship is an EU initiative that "aims to secure a major role for Europe in the ongoing technological revolution, helping to bring graphene innovation out of the laboratory and into commercial applications by 2023” (see MRS Bulletin, doi:10.1557/ mrs.2017.14).

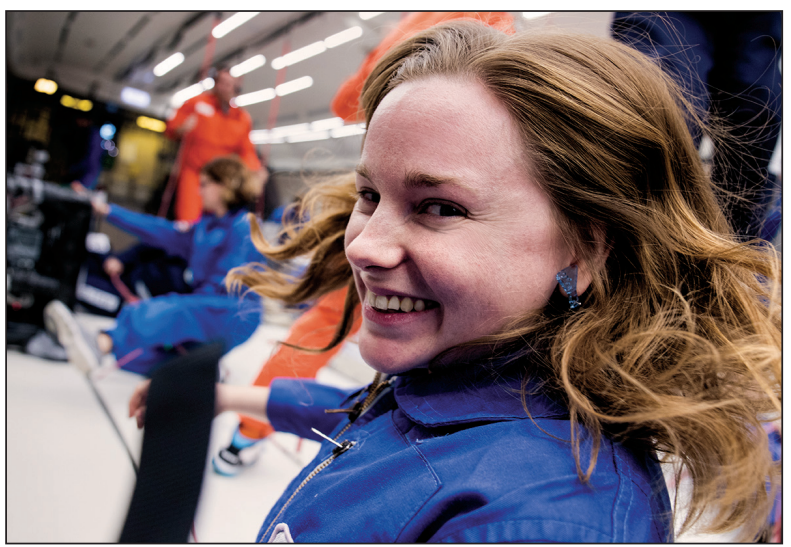

In May 2019, the Graphene Flagship embarked on a campaign of zero-gravity parabolic flights to test novel thermal management graphene devices for space applications. The unique properties of graphene can significantly improve the performance of the devices. Credit: Graphene Flagship.
The showcase set out to demonstrate that graphene is more than a laboratory novelty. Frank Koppens, chair of the Graphene Flagship's MWC Committee, says that the idea was to demonstrate that "now it is time for graphene to be moved from the laboratory onto the factory floor."

The Graphene Flagship was launched as an initiative in 2017 with a heavy emphasis on "market-motivated, industry-led targeted sub-projects." The yardstick for these projects is their progress in improving the "technology readiness level," a term devised by NASA. The "TRL" is a widely used measure for benchmarking the maturity of a technology.

Confirming that graphene really does have commercial potential is important to avoid the fate of other recent materials "breakthroughs," such as high-temperature superconductors and $\mathrm{C}_{60}$ (buckyball), which failed to transition from academic research papers to products and profits. Both materials made newspaper headlines, and, like graphene, collected Nobel prizes, but with little apparent commercial impact. The Graphene Flagship, now halfway through its 10 -year program, is a concerted effort to avoid this fate. As Jari Kinaret, director of the Graphene Flagship, puts it, the initiative has an "overarching goal of taking graphene from academic laboratories to society."

The Flagship now uses the phrase "graphene and related materials" or GRMs, to reflect the fact that it now supports R\&D in other two-dimensional (2D) materials beyond graphene. For example, the Flagship has also backed work on bilayers of graphene and boron nitride and on 2D silicon.

The Flagship operates through a series of "work packages." One part of this activity, aimed specifically at industrialization, sets out to convince potential commercial users that graphene is a well-characterized and well-understood material, and not a laboratory curiosity. To this end, the Flagship operates a "validation service," which draws on authorized national measurement institutes and facilities in Europe. The validation service started last fall and, according to Alexander Tzalenchuk of the UK's National Physical Laboratory, "we had been slightly concerned whether it would be in demand." It turns out that the concerns were unfounded, with more than 30 requests so far. "The kind of requests that we get range from simple confirmation of what a company, or a research group for that matter, has measured internally or have learnt from their suppliers, to more elaborate problem solving," says Tzalenchuk, who leads the validation activity.

The Flagship is building on the validation activity to develop standards for GRMs. As the Flagship's latest review puts it, "Industrial players need standardized materials and processes in order to integrate these new materials and devices into their innovation activities." As a part of this work, the Flagship has set up a standardization committee and is working with international standardization bodies, such as the International Electrotechnical Commission (IEC) in its work on nanotechnology for electrical, electronic, and related products and systems.

The Flagship also addresses manufacturing challenges. It plans to create an experimental pilot line for graphenebased electronics, optoelectronics, and sensors. As a part of this work, the Flagship has put out a call for a partner who can bring "specific industrial and technology transfer competencies or capabilities" to collaborate on sustainable production of GRMs.

Alex Jouvray, program manager for Nanoinstruments at Aixtron Ltd., says that the Flagship's production activity 
is "entirely driven by industry, and the material and equipment producers have to adhere to strict development and quality control protocols."

"Our ultimate goal," he adds, "is to establish an industrial graphene supply chain in Europe that can support the diverse applications of graphene."

The Flagship has cast its net wide to attract industrial collaborators. The Composites Work Package, for example, includes Airbus, Fiat, ABB, and BASF, among its industrial partners.

Leonardo, a major aerospace and defense company, is the industrial partner in a recent series of zero-gravity parabolic flights to test novel thermal management graphene devices for space applications. The company is working on a graphene loop heat pipe that could play a role in thermal management for satellites. The idea is that graphene will improve the heat transfer to cool electronic devices and instruments.

The Flagship took another small step into space in June of this year, with the launch of a sounding rocket to test the printing of graphene devices in space. The Materials Science Experiment Rocket
(MASER) 14 was launched from the Essrange Space Center in Sweden in collaboration with the European Space Agency. According to the Flagship's announcement, "The objective is to test the printing of graphene patterns on silicon substrates in zero-gravity conditions. This mission is also a first step toward the investigation of graphene for radiation shielding purposes, an essential requirement of manned space exploration."

Where the parabolic flights enabled 24 seconds of microgravity, the flight of the MASER 14 rocket enabled more than six minutes of microgravity. Carlo Ilorio, leader of the Flagship's space activities, and a researcher at the Université libre de Bruxelles, says, "There is no better way to validate graphene's potential than to send it to the environment it will be used in."

As well as working with large businesses, the Flagship supports startup companies. The latest review put the tally at nine spin-offs. For example, BeDimensional S.p.A., set up in 2016, produces 2D crystals on an industrial scale. In 2018, the company, a spin-off of the Istituto Italiano di Tecnologia, a Flagship member, announced a private investment of $€ 18$ million. Kari Hjelt, head of Innovation of the Graphene Flagship, says the investment "exemplifies the growing interest of investors in graphene applications. We are moving from technology push toward market pull and the promise of graphene to transform many application fields."

Atomic Mechanics, another associated member of the Graphene Flagship, founded in Manchester in 2017, works on a range of sensor and actuator devices based on patented graphene technology.

One of Europe's earlier graphene startups, GrapheneTech, an associated member of the Graphene Flagship, has been making graphene since 2010. José Antonio Peláez, the Spanish company's director of Operations, recently pointed out the challenge that faces potential users of the material. "Once we were able to produce graphene, we realized that companies didn't know how to use it," he said in a recent interview. This prompted GrapheneTech to move beyond producing graphene powder as a raw material and develop applications, in particular for energy storage, additives for polymers, lubricants and greases, and coatings and inks.

Michael Kenward
South Africa builds on hydrogen fuel-cell program

hysasystems.com

$\mathrm{T}$ here is growing interest internationally in the use of hydrogen fuel cells (HFCs) as an alternative source of energy. Besides their potential for powering homes and buildings, hydrogen fuel cells can be used in transportation applications to power forklifts, cargo trucks, delivery vans, and passenger vehicles. Their benefits include higher efficiency when compared to diesel, environmental friendliness (zero exhaust emissions), noise-free mobility, and lower maintenance (no oil changes).

HFC technologies topped the agenda at the 2019 Hydrogen and Fuel Cells Program Annual Merit Review and Peer Evaluation Meeting held in Virginia last spring. Convened annually by the US Department of Energy (DOE), the meeting reviews $\mathrm{HFC}$ projects funded by DOE's Hydrogen and Fuel Cells Program.

Among the participants at this year's meeting were Phil Mjwara, the DirectorGeneral of South Africa's Department of Science and Technology (DST), and the DST's Chief Director for Hydrogen and Energy, Rebecca Maserumule.

The DST, through its Hydrogen South Africa (HySA) program, has made significant progress with local applications of HFC technology. In 2016, in partnership with Impala Refining Services and the University of the Western Cape, the DST/HySA unveiled a prototype hydrogen fuel cell forklift and refueling station in Springs, Gauteng.

The DST and HySA are also piloting a number of other prototypes and demonstrators, including a standby HFC power plant at Poelano High School in South Africa.

Keith Schmid, Chief Operating Officer of US company Plug Power, reported at the meeting that customer acceptance of HFCs was increasing. "We have experienced significant advances in commercial fleet vehicle applications," he said.

From South Africa, Mjwara and Maserumule met with representatives of Plug Power on the sidelines of the meeting. According to Mjwara, the DST is exploring the possibility of partnering with Plug Power, which works in various areas of HFC application. Plug Power designs and manufactures HFC systems that replace conventional batteries in equipment and vehicles powered by electricity. According to the DST, the company has had considerable success in the deployment of hydrogen refueling stations aimed at accelerating the adoption of HFC solutions. 